Gut, 1976, 17, 517-526

\title{
Permeability of the small intestine after intra-arterial injection of histamine-type mediators and irradiation
}

\author{
J. G. C. KINGHAM AND C. A. LOEHRY \\ From the Department of Medicine, Royal Victoria Hospital, Boscombe, Bournemouth
}

SUMMARY Permeability and selectivity of rabbit small intestine were estimated by a perfusion technique after intra-arterial injection of histamine-type mediators and intestinal irradiation. It was shown that the histamine-type mediators caused an increase in capillary permeability which produced an overall moderate increase in transmucosal permeability with a moderate loss of selectivity. Local intestinal irradiation caused a very marked increase in permeability and a profound loss of selectivity. It was felt that this was produced partly by an increase in capillary permeability but largely by damage to the epithelial basement membrane. It is concluded that the intestinal capillary endothelium is both rate-limiting and selective, though not to a major degree in either case. The epithelial basement membrane, however, appears to be both rate-limiting and markedly selective.

The purpose of studying the permeability and selectivity of the small intestinal mucosa under experimental conditions is to gain information about the mechanisms of exudative enteropathies. Exudative enteropathies usually manifest themselves clinically by excessive enteric protein loss. It is well recognised that the small intestine is very highly selective as regards its exudation (Loehry et al., 1970 ), and that in health only minimal quantities of protein are lost into the intestinal lumen. Thus it must be assumed that in a protein losing enteropathy, where sometimes up to 50 times the normal amount of plasma protein is being lost into the intestine (Waldmann, 1972), there is an associated loss of normal mucosal selectivity. If this were not the case then the patient with a protein losing enteropathy would be very rapidly depleted of the smaller plasma solubles.

It is therefore important to know which of the intestinal barriers are responsible for mucosal selectivity. In the preceding paper (Kingham and Loehry, 1976a) the three main anatomical barriers to intestinal sorption were briefly discussed, and one of them, the epithelial cell layer, was investigated and shown to be highly impermeable - that is, rate limiting-but non-selective. In this paper we studied the effects on overall mucosal permeability and selectivity of damage to the epithelial basement membrane and the capillary endothelium.

Received for publication 7 April 1976
Histamine-type mediators were used to increase mucosal capillary permeability. It has been shown by many investigators (Majno et al., 1961; Majno and Palade, 1961; Clementi and Palade, 1969a, b; Gabbiani et al., 1970), that histamine and bradykinin increase capillary permeability primarily by disruption of the endothelial cell sheet, thus allowing intercellular leakage, and partly by removal of the fenestral diaphragms affording transcellular leakage.

The procedure chosen to produce damage to the epithelial basement membrane was local intestinal irradiation. The basis of this choice is that irradiation has been shown to produce alveolar basement membrane damage when directed to the lung (Koshurnikova et al., 1972, 1973), and glomerular basement membrane damage with non-selective proteinuria in radiation nephritis (Madrazo et al., 1969; Gang et al., 1973). Furthermore, radiation enteritis may result in a protein losing enteropathy in patients undergoing radiotherapy for pelvic cancer (Löbe and Schneider, 1967; Birke et al., 1967), and it also produces leakage of plasma macromolecules into the intestine of experimentally irradiated animals (Vatistas and Hornsey, 1966).

\section{Methods}

The basic perfusion technique for estimation of small intestinal permeability and selectivity has been described in the preceding paper. The principle of the method is to measure the exsorption of various 
tracer substances $\left({ }^{125} \mathrm{IPVP},{ }^{3} \mathrm{H}\right.$ inulin, ${ }^{57} \mathrm{Co}$ vitamin $B_{12}$, creatinine, and urea) from plasma to intestinal lumen in both experimental and control situations, and to compare the results. Various modifications were made to adapt the technique to assess the effects of local histamine-type mediators and irradiation.

INTRA-ARTERIAL INJECTION OF HISTAMINE TYPE MEDIATORS

Two equal lengths of rabbit small intestine were isolated, cannulated, and perfused with standard perfusate. The loops were chosen so that the blood supply to each was by one main arterial radicle.

After an equilibration period, two 25 gauge intravenous butterfly needles, primed with normal saline, were inserted one into each of the arterial radicles supplying the loops. The loops were carefully handled and positioned to ensure that the needles neither ruptured the arteries nor obstructed the blood flow through them more than could be avoided.

Five millilitres of normal saline were slowly injected into one of the arteries while perfusing the loop with normal perfusate at a rate of $2 \mathrm{ml} / \mathrm{min}$. Samples of perfusate were collected at five minute intervals for 20 minutes. The perfusates collected during this part of the experiment were used as controls. Plasma samples were taken at the start and finish of the perfusion.

After this control period $5 \mathrm{ml}$ of either histamine acid phosphate $(0.5 \mathrm{mg} / \mathrm{ml})$ or bradykinin triacetate $(0.1 \mathrm{mg} / \mathrm{ml})$ were slowly infused over 20 minutes into the other cannulated artery and an additional series of four five-minute samples collected; the rate of perfusion remained at $2 \mathrm{ml} / \mathrm{min}$. As soon as the last perfusate sample had been collected, $5 \mathrm{ml}$ Pelikan carbon black suspension were injected intravenously into the animal in order to assess capillary permeability by carbon labelling. Fifteen minutes after this injection a biopsy of the experimental loop was taken and processed for histological examination. In order to get histological control material, the carbon administration was performed immediately after the control perfusion in four of the animals and the loop subsequently subjected to biopsy.

\section{SMALL INTESTINAL IRRADIATION}

The rabbits underwent a preliminary operation under full aseptic conditions to irradiate a segment of the small intestine. A $3 \mathrm{~cm}$ midline longitudinal incision was made midway between the xiphisternum and the pubis with the rabbit lying supine. A loop of small intestine was withdrawn from the abdomen through the incision and followed either proximally to the duodenum or distally to the tip of the appendix in order to gain orientation. A $30 \mathrm{~cm}$ distal jejunal or proximal ileal loop was marked out by two fine $(6 / 0)$ silk sutures inserted into the mesentery close to the intestine. Care was taken not to impair the blood supply to any part of the small bowel by this procedure. All the intestine, apart from the marked loop, was replaced in the abdominal cavity and the animal turned onto its side. The exteriorised loop of gut was supported on a raised platform in order to isolate it as far as possible from the rest of the animal while still maintaining its blood supply. The whole of the animal apart from the exteriorised loop was shielded with lead. The loop was given $1500 \mathrm{r}$ of gamma irradiation over 20 minutes from a TEM Cobalt 60 source.

After irradiation the marked loop was replaced in the peritoneal cavity and the abdominal incision repaired in layers. Apart from the production of a thin serous exudate the irradiated loops did not appear abnormal at the time of replacement in the abdomen.

The animals quickly recovered after the procedure and were eating and drinking normally by the following morning.

Six days after irradiation the definitive perfusion was performed. This involved the cannulation and perfusion of the marked irradiated segment and an adjacent segment of non-radiated small bowel to act as control. Perfusates were collected at 15 minute intervals for one hour and plasma samples taken at the start and finish of the procedure.

When the perfusion was completed biopsies of both loops were taken for histological examination. In six of the rabbits carbon black was injected intravenously as in the histamine experiments in order to assess capillary permeability.

In all experiments overall mucosal permeability was measured by estimation of the exsorbed plasma tracer substances in the perfusates, and overall mucosal selectivity by fractionation of the PVP in the perfusates and plasma.

\section{HIST AMINE}

The effects on the permeability of perfused small bowel loops produced by local intra-arterial injection of either normal saline (control) or histamine are shown in Fig. 1. The results are expressed as clearances of each tracer substance into control and experimental loops. Table 1 shows the average clearances of the tracer substances into the control and histamine-injected loops, and also expresses the results as a percentage increase in clearance for each tracer substance induced by histamine.

It is evident from Fig. 1 and Table 1 that local intra-arterial histamine significantly increases the overall mucosal permeability to substances greater in 


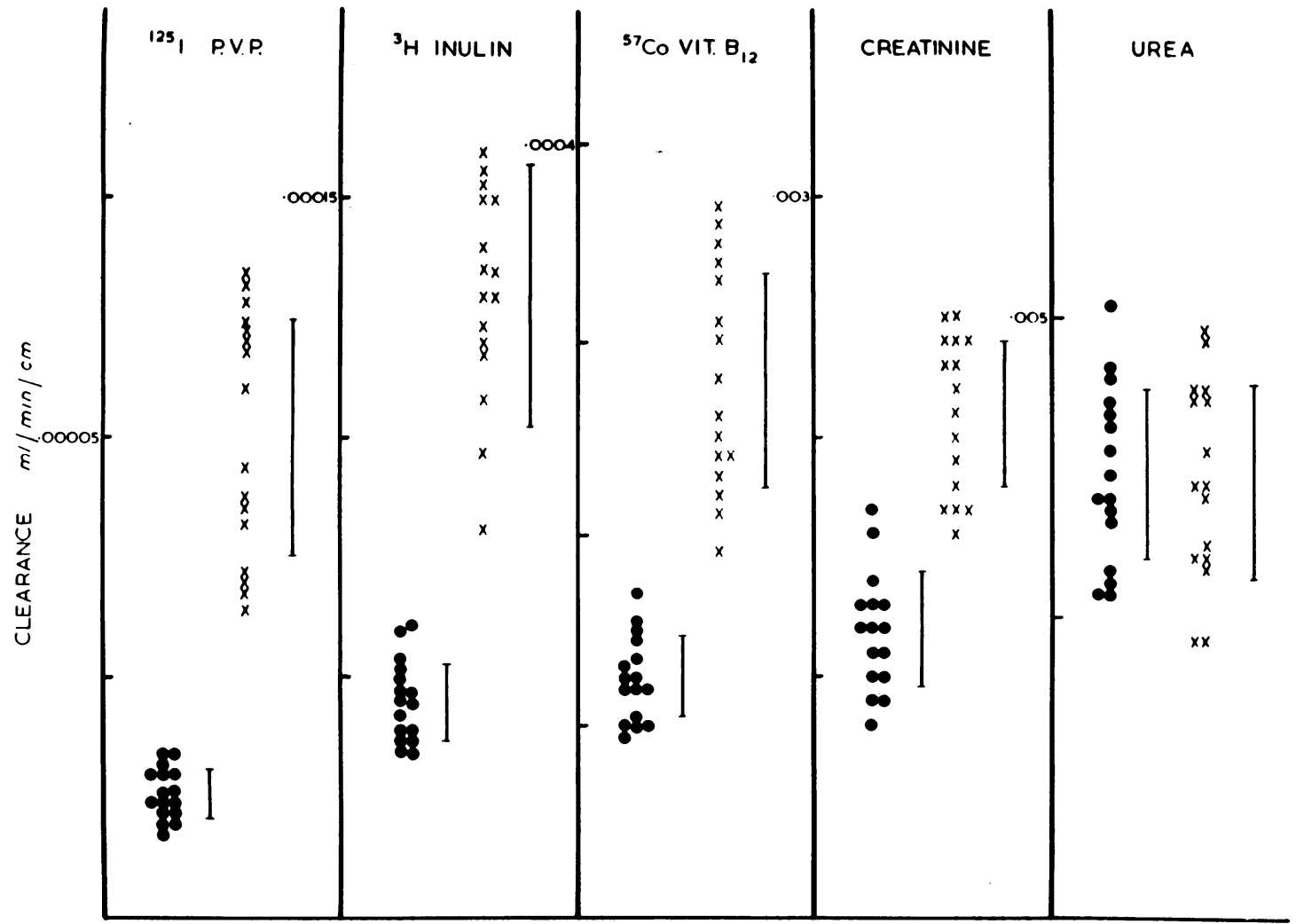

Fig. 1 The changes in permeability of the small intestine produced by local intra-arterial injection of either normal saline (control $\bullet$ ) or histamine $(X)$. The results are expressed as clearances $(\mathrm{ml} / \mathrm{min} / \mathrm{cm})$ of each tracer substance into the control and experiment loops.

size than urea. It also appears that the greater the molecular size of the tracer substance, the greater the increase in permeability-in other words, histamine has reduced the selectivity of the mucosal barriers.

In order to substantiate this loss of selectivity the fractionated PVP clearances curves for the control loop and the histamine-treated loop were compared (Fig. 2). It may be seen that the PVP exudation into the histamine loop is less selected than that into the control loop as shown by the flatter histamine curve.

\section{HISTOLOGY}

Figure $3 \mathrm{a}$ and $\mathrm{b}$ shows the light microscopical appearances of the control and histamine-treated loops stained with neutral red to demonstrate carbon particles.

The sections of the histamine-treated loops show a widening of the lamina propria of the villous core due to oedema, and marked labelling of the capillaries with carbon. This denotes a great increase in capillary permeability over the control loop. There was no obvious increase in cellularity of the villous

Table 1 Average clearances (with SD from means) of each tracer substance into control and histamine injected loops

\begin{tabular}{|c|c|c|c|c|c|}
\hline \multirow[t]{2}{*}{ Tracer substance } & \multirow[t]{2}{*}{$M W$} & \multicolumn{2}{|c|}{ Mean clearance $\pm S D(\mathrm{ml} / \mathrm{min} / \mathrm{cm})$} & \multirow[t]{2}{*}{$S D$ P } & \multirow[t]{2}{*}{$\%$} \\
\hline & & Control & Histamine & & \\
\hline $\begin{array}{l}\text { PVP } \\
\text { Inulin } \\
\text { Vitamin } \mathbf{B}_{12} \\
\text { Creatinine } \\
\text { Urea }\end{array}$ & $\begin{array}{r}33000 \\
5000 \\
1300 \\
120 \\
60\end{array}$ & $\begin{array}{l}0.000013 \pm 0.000002 \\
0.000045 \pm 0.000008 \\
0.00013 \pm 0.00002 \\
0.0012 \pm 0.0002 \\
0.0037 \pm 0.0007\end{array}$ & $\begin{array}{l}0.000050 \pm 0.000012 \\
0.000130 \pm 0.000027 \\
0.00028 \pm 0.00006 \\
0.0021 \pm 0.0003 \\
0.0036 \pm 0.0008\end{array}$ & $\begin{array}{l}<0.001 \\
<0.001 \\
<0.001 \\
<0.001 \\
>0.8\end{array}$ & $\begin{array}{r}290 \\
190 \\
120 \\
80 \\
-3\end{array}$ \\
\hline
\end{tabular}

The results are also expressed as a percentage increase in clearance for each tracer substance induced by histamine. 


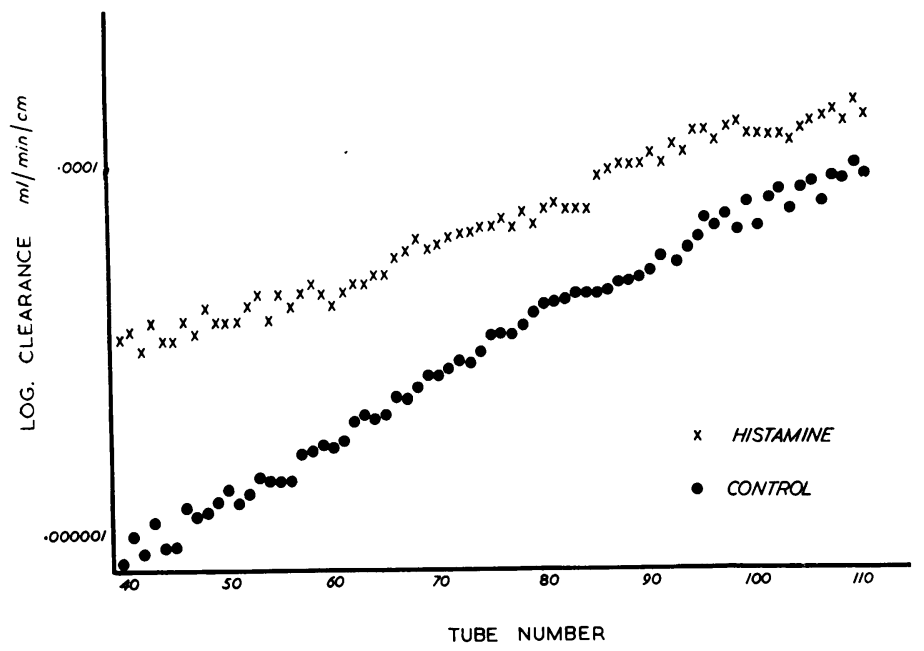

Fig. 2 The clearances into the histamine-injected and control loops of the individual fractions of PVP as they came off the column plotted against the tube number. The tube number corresponds to a particular molecular weight which is known from the calibration of the column.
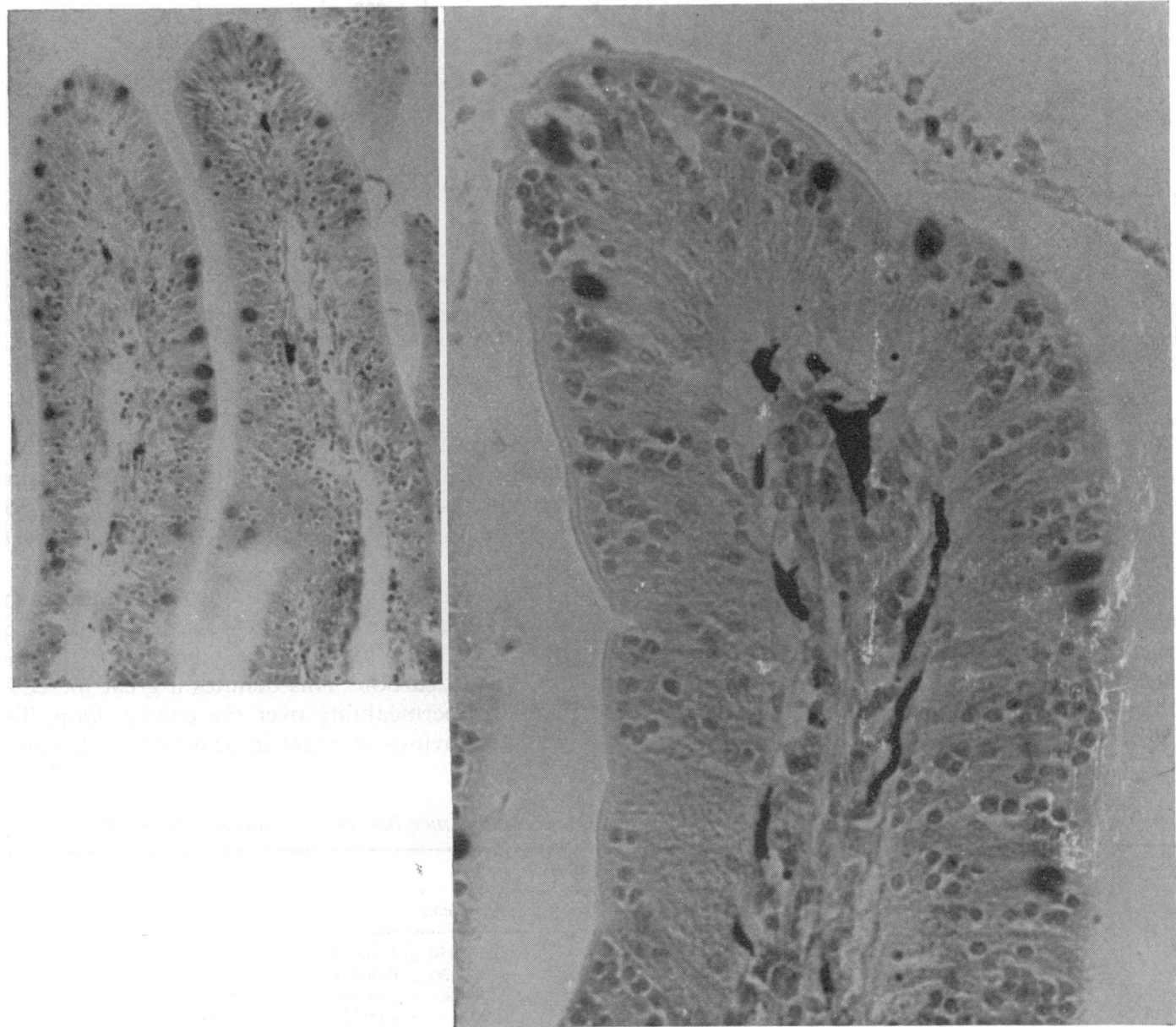

(a)

(b)

Fig. 3 Light microscopical appearances of the control $(a)$ and histamine treated $(b)$ loops stained with neutral red to show carbon particles. 
core. The epithelium and its basement membrane appeared normal with an intact brush border and intercellular junctions.

The results of the bradykinin experiments were qualitatively very similar to those of the histamine experiments, although the absolute increase in capillary permeability was slightly greater using bradykinin.

\section{Discussion}

\section{HISTAMINE EXPERIMENTS}

The capillary endothelium, under normal conditions, is an unbroken barrier between the vascular lumen and the extravascular space. This barrier allows restricted passage of the plasma contents out into the extravascular space. Pappenheimer has shown that the capillary endothelium is a selective barrier which limits the permeation rates of plasma solutes according to their molecular sizes. There is an inverse linear relationship between the molecular weight of a water soluble substance and its permeability coefficient across the capillary wall (Pappenheimer et al., 1951; Grotte, 1956; Mayerson et al., 1960). This selective nature of the capillary endothelium only applies to molecules below a certain molecular weight, approximately $60000-100000$. Above this molecular weight the capillary endothelium behaves purely as a non-selective rate limiting barrier to molecules up to molecular weight 400000 and greater (Mayerson et al., 1960). To explain this on the basis of the pore theory it is necessary to propose that there are two sets of aqueous pores; (1) the major series of small pores with a radius of $30 \mathrm{~A}^{\circ}$ allowing restricted passage of molecules up to the size of albumin or a little larger; (2) a smaller system of large pores of radius $\mathrm{A}^{\circ}$ to allow free passage of molecules up to the size of high molecular weight dextrans and ferritin. These estimated pore radii are only approximations, but Pappenheimer et al. (1951), Grotte (1956), and Clementi and Palade $(1969 \mathrm{a}, \mathrm{b})$ all agreed with the two size pore system with similar estimates for pore size.

The effect of histamine type mediators on capillary permeability has been extensively studied in recent years, mainly by macroaggregate and macromolecule labelling techniques. The labelling substances most often used are carbon black suspension, colloidal mercuric sulphide, colloidal gold, and ferritin, the molecular sizes of which range from 70-500 $\mathrm{A}^{\circ}$. The basis of the technique is that under normal conditions there is practically no leakage of these intravenously administered tracers out of the intravascular compartment. Under conditions of increased capillary permeability the tracers can leak out of the vascular lumen to rest against the capillary basement membrane and so label the permeable vessels.
The work of Majno et al. (1961, 1967), Clementi and Palade (1969a, b), and Gabbiani et al. (1970) on in vivo capillary preparations has shown that histamine type mediators increase capillary permeability. The mode of action is not fully established but histamine and bradykinin probably exert their effect partly by contraction of the endothelial cells producing intercellular gaps, and partly by removal of the diaphragms from the fenestrae of the endothelial cell membrane allowing transcellular leakage. In either event macromolecules can pass through the endothelium to come to rest on the intact endothelial basement membrane.

In the present study it can be seen from the results of the perfusion experiments that histamine-type mediators cause a moderate increase in overall mucosal permeability concomitant with the increase in capillary permeability. The percentage increment in permeation rate for each tracer substance was proportional to its molecular size, the PVP clearance was affected the most and creatinine clearance the least. Urea clearance was unaffected.

These figures indicate, therefore, that a decrease in mucosal selectivity has occurred; this is borne out by the clearance curves of fractionated PVP. The clearance curves of the histamine and bradykinin treated loops are much flatter than the control curves, denoting a marked loss of selectivity. The slopes of the clearance curves are as follows:

$\begin{array}{ll}\text { Histamine: } & 0.021 \pm 0.003 \\ \text { Bradykinin: } & 0.020 \pm 0.003 \\ \text { Control: } & 0.033 \pm 0.002\end{array}$

The difference between the PVP slopes of bradykinin and histamine treated loops is not significant ( $P>0.60$ ), but both the treated loops differed from the control highly significantly $(P>0.001)$.

The absolute increases in permeability after histamine type mediators were only moderate, the greater being after bradykinin.

Thus, histamine and bradykinin produce an overall increase in mucosal permeability associated with a loss of selectivity.

The histological results show an increase in capillary permeability without obvious light microscopical changes in the epithelium or its basement membrane. It is suggested that these vasoactive substances exert their effects on overall mucosal permeability by increasing exudation in a nonselective fashion from the capillaries. This, in turn, creates an increased concentration gradient from interstitium to intestinal lumen which is reflected by the increase in plasma to lumen clearance of the tracer substances.

\section{Irradiation}

Figure 4 demonstrates the early changes in perme- 


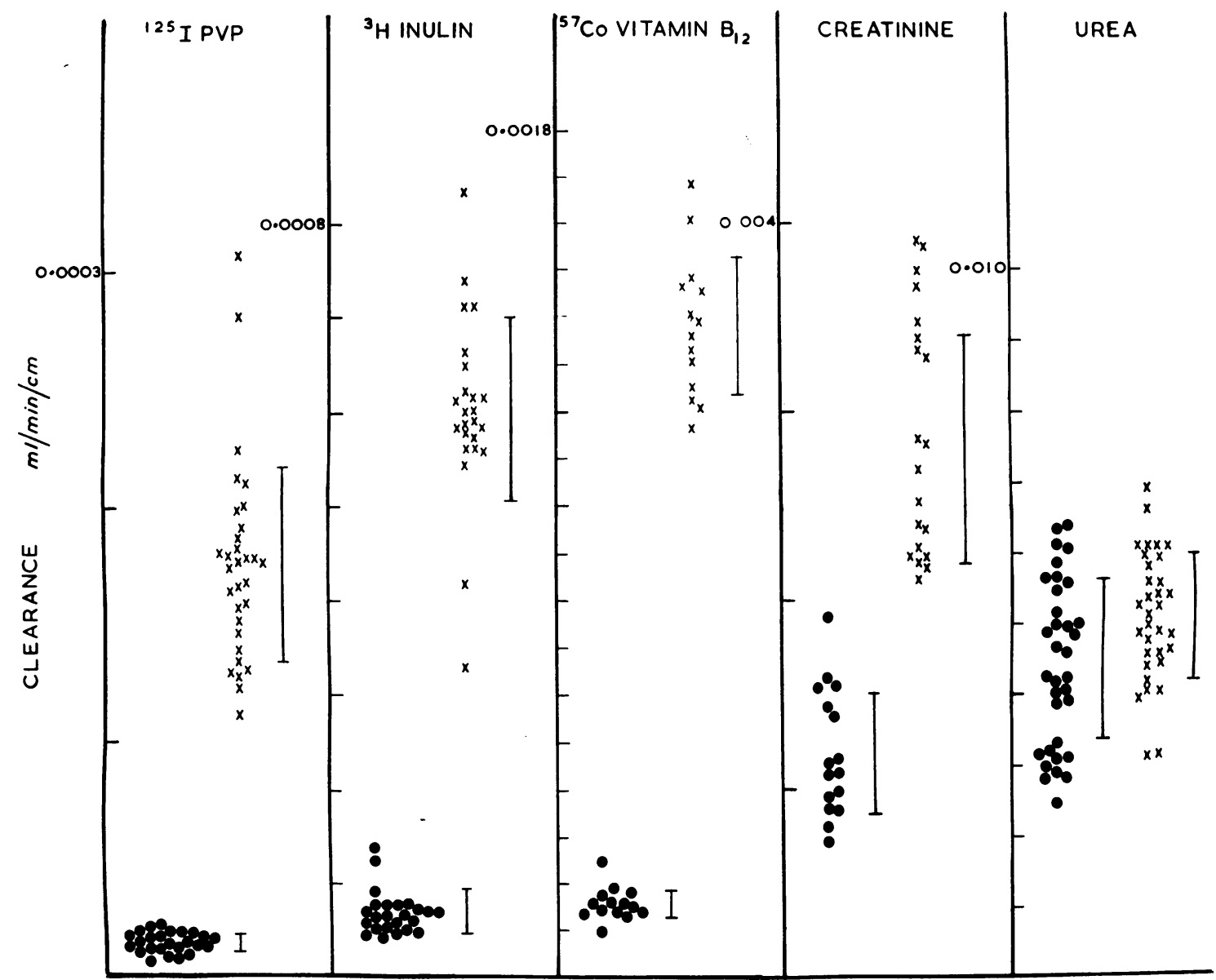

Fig. 4 The changes in permeability of the small intestine six days after irradiation shown by comparison of the clearances of the tracer substances into control $(\bullet)$ and irradiated (six days) loops $(\times)$.

ability of the small intestine induced by radiation after six days by comparing the clearance of the tracer substances into the control and irradiated segments. Table 2 shows the average clearances, with standard deviations, of each of the tracer substances into irradiated and control loops and expresses the results as a percentage increase in clearance induced by radiation.
It is clear from Fig. 4 and Table 2 that there was an increase in permeability to all substances tested except urea, the greatest increase being for the largest tracer, PVP, and the smallest increase for creatinine. The larger the molecular weight of the tracer substance, the greater was the increase in its permeation rate.

From Table 2 and Fig. 4 it is obvious that the

Table 2 Average clearances (with SD) of each tracer substance into control and irradiated loops

\begin{tabular}{|c|c|c|c|c|c|}
\hline \multirow[t]{2}{*}{ Tracer substance } & \multirow[t]{2}{*}{$M W$} & \multicolumn{2}{|c|}{ Mean clearance $\pm S D(\mathrm{ml} / \mathrm{min} / \mathrm{cm})$} & \multirow[t]{2}{*}{$S D \mathbf{P}$} & \multirow[t]{2}{*}{$\%$} \\
\hline & & Control & Irradiation 6 days & & \\
\hline $\begin{array}{l}\text { PVP } \\
\text { Inulin } \\
\text { Vitamin } \mathbf{B}_{\mathbf{1 2}} \\
\text { Creatinine } \\
\text { Urea }\end{array}$ & $\begin{array}{r}33000 \\
5000 \\
1300 \\
120 \\
60\end{array}$ & $\begin{array}{l}0.000014 \pm 0.000004 \\
0.000059 \pm 0.000023 \\
0.00016 \pm 0.00003 \\
0.0012 \pm 0.0003 \\
0.0045 \pm 0.0011\end{array}$ & $\begin{array}{l}0.000176 \pm 0.000041 \\
0.000602 \pm 0.000097 \\
0.00138 \pm 0.00014 \\
0.0028 \pm 0.0006 \\
0.0051 \pm 0.0009\end{array}$ & $\begin{array}{l}<0.001 \\
<0.001 \\
<0.001 \\
<0.001 \\
>0.05\end{array}$ & $\begin{array}{r}1120 \\
920 \\
780 \\
140 \\
10\end{array}$ \\
\hline
\end{tabular}

The results are also expressed as a percentage increase in clearance after irradiation. 


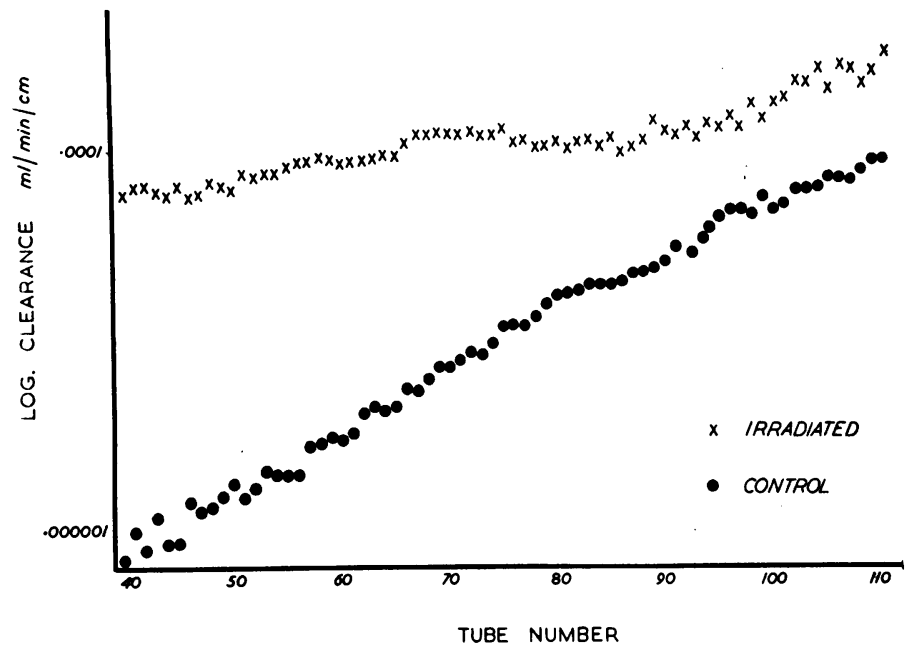

Fig. 5 The clearances into the control and irradiated loops of the individual fractions of PVP as they came off the column. The clearances are plotted against tube number where tube number represents a particular molecular weight known from calibration of the column.

effect of radiation on permeability to the various tracers was not uniform. There was a very great increase in permeability to the largest tracers but progressively less effect in decreasing order of size of the tracers. Thus irradiation preferentially increases the permeability of small intestine to larger molecules -that is, the increase in permeability is associated with a very marked loss of selectivity.

This loss of selectivity is confirmed by fractionation of the PVP. Figure 5 shows the clearances into the control and irradiated loops of the individual fractions of PVP. The slope of the clearance curve of the irradiated loop is much less steep than the control curve. This means that after irradiation there is less difference between the clearance of the high and low molecular weight fractions of PVP. Therefore there is a marked increase in PVP permeability with a profound loss of selectivity six days after irradiation.

\section{Histology}

Figure $6 \mathrm{a}$ and $\mathrm{b}$ shows sections of the irradiated and control loops stained with haematoxylin and eosin. It can be seen that the villi of the irradiated loop are slightly shorter with an increase in the thickness and cellularity of the lamina propria. However, the epithelium itself is intact with no separation or lifting of the cells from their basement membrane, and a healthy looking brush border and lateral cell membranes.

The sections from the animals which had been injected with carbon showed that there was a slight increase in capillary permeability of the irradiated loop compared with the control demonstrated by a greater degree of carbon labelling.

\section{IRRADIATION EXPERIMENTS}

Exposure of the gastrointestinal tract to ionising radiations causes morphological damage to the intestinal mucosa, and an increase in passive permeability to proteins and other macromolecules.

It has been observed by both Sullivan (1961) and Vatistas and Hornsey (1966) that histological damage to the intestinal epithelium does not correlate with macromolecular leakage. The morphological damage was maximal at 12-72 hours after irradiation, while macromolecular leakage did not start until after 72 hours. Moreover, the macromolecular leakage remained high until day 8 or 9 , by which time the morphological damage had apparently completely recovered.

Willoughby (1960, 1961, 1962), Sullivan (1961), and Vatistas and Hornsey (1966), consider that irradiation-induced intestinal protein loss is secondary to an increase in capillary permeability. Only Willoughby, however, offers any evidence to support his theory. He noted an increased tissue content of intravenously administered tryptan blue in irradiated intestine compared with control intestine.

In the present study the permeability characteristics of the small intestine were observed at six days after irradiation because it has been consistently shown that macromolecular loss should be at its peak by this time.

There was a very marked increase in small intestinal permeability, and the percentage increment in 


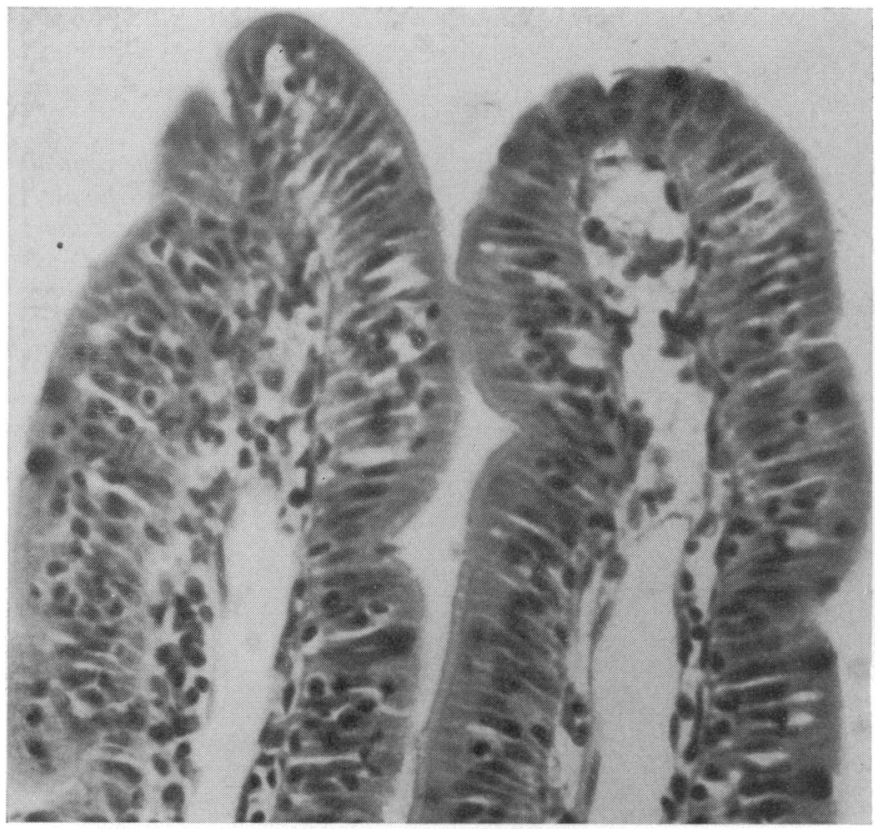

(a)

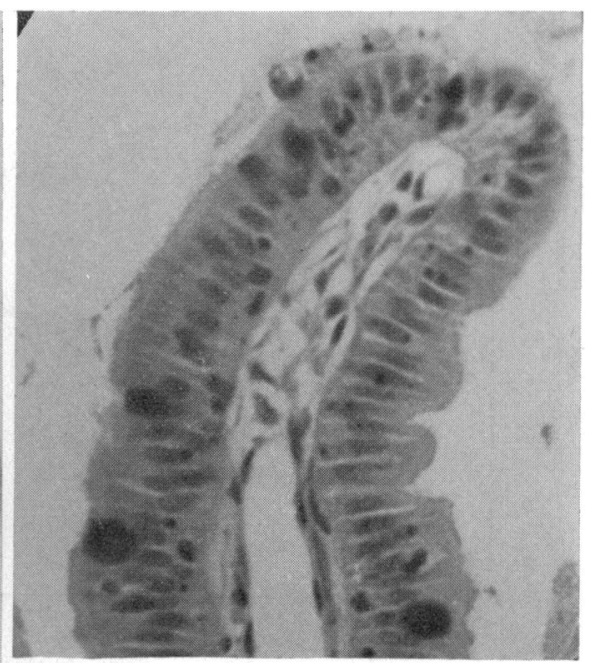

(b)

Fig. 6 Light microscopical appearance of the irradiated $(a)$ and control (b) loops stained with haematoxylin and eosin.

permeation rate after irradiation, for each tracer substance increased with molecular weight. The only exception to this was urea, for which substance there was no increase in permeability after irradiation. These results denote a loss of mucosal selectivity, and this is, in fact, borne out by the clearance curve of fractionated PVP. The curve for irradiated small intestine is much flatter than that of the control: the slope of the irradiated bowel clearance curves is $0.011 \pm 0.002$ and of the controls $0.033 \pm 0.002$.

The histological results of the present study reveal an almost normal mucosa with a slight shortening and thickening of the villi as the only obvious change. The epithelial cells themselves appear completely normal. These observations are in good agreement with those of Trier and Browning (1966) and Wiernik and Plant (1970). Indeed, from first principles, it would be expected that the villous cell population would be normal six days after a single dose of irradiation because all these villous cells must be newly produced from the stem cells of the crypts during the 'overshoot period' of proliferative cellularity three to four days after irradiation.

The carbon labelling techniques have shown that there is a moderate increase in capillary permeability after irradiation, which shows that an increase in vascular permeability may be an essential factor in the response of the small intestine to irradiation. However, irradiation did not produce such a marked increase in vascular permeability as histamine or bradykinin. Therefore, increased capillary permeability alone is unlikely to be responsible for the very marked increase in mucosal permeability to macromolecules demonstrated after irradiation, although it may partly account for the loss of selectivity. However, there is considerable difference in the selectivity of irradiated and histamine treated bowel, the irradiated bowel being much less selective.

Slope of PVP curve-irradiation:

$$
0.011 \pm 0.002
$$$$
P<0.001
$$

Slope of PVP curve-histamine:

$$
0.021 \pm 0.003 \quad P<0.001
$$

On the results of these experiments it seems unlikely that the mucosal permeability induced by irradiation is caused by epithelial damage or solely mediated by increased capillary permeability.

It is suggested that the profound loss of selectivity was caused by epithelial basement membrane damage, although there is no direct evidence to support this theory. Some measure of indirect support comes from work in other fields; Koshurnikova et al. (1972, 1973) found thickening of the alveolar basement membrane after irradiation of the lung, and irradiation of the kidney produces glomerular 
basement membrane damage associated with nonselective proteinuria (Madrazo et al., 1969; Gang et al., 1973).

On the clinical side basement membrane abnormalities are well recognized in diseases associated with an increase in intestinal permeability such as coeliac disease (Shiner, 1967), collagenous sprue (Weinstein et al., 1970), and tropical sprue (Mathan et al., 1975).

The changes in the physical properties of the mucosal barriers after irradiation may be conjectured in the following manner: there is a moderate increase in the size of the capillary endothelial pores and a large increase in the size of the epithelial basement membrane pores. The epithelial cell pores are unaltered. Thus the irradiation-damage intestine affords an increase in permeability and a loss of selectivity.

\section{Overall conclusions}

An increase in capillary permeability induced by histamine and bradykinin causes a moderate increase in mucosal permeability and a moderate loss of macromolecular selectivity.

Irradiation produces a slight increase in capillary permeability but the overall effect is a great increase in mucosal permeability and a profound loss of macromolecular selectivity which we believe may be secondary to basement membrane damage.

The factors governing the permeability of the intestinal barriers to large molecules are not the same as those for small molecules. It is probable that the normal mucosa is nearly maximally permeable to a substance as small as urea, and that permeation in these cases is governed by the unstirred water layers.

Thus the intestinal barriers all have different permeability characteristics. It is proposed that the epithelial cell layer is highly rate limiting and nonselective, the capillary endothelium is selective and moderately rate limiting, and the epithelial basement membrane is highly selective and moderately rate limiting, though at present the proposal remains speculative.

\section{References}

Birke, G., Jacobsson, F., Liljedahl, S. O., Plantin, L. O., and Wetterfors, J. (1967). Catabolism of albumin and gamma globulin after treatment with ionising radiation to the abdomen. Acta Radiologica Therapy Physics Biology, 6, 113-121.

Clementi, F., and Palade, G. E. (1969a). Intestinal capillaries, 2: structural effects of EDTA and histamine. Journal of Cell Biology, 42, 706-714.

Clementi, F., and Palade, G. E. (1969b). Intestinal capillaries: 1: permeability to peroxidase and ferritin. Journal of Cell Biology, 41, 33-58.
Intra-arterial injections of histamine, serotonin or bradykinin: a topographic study of vascular leakage. Proceedings of the Society for Experimental Biology and Medicine, 135, 447-452.

Gang, N. F., Sarophim, M. E., Madrazo, A., and Churg, J. Radiation nephritis. American Journal of Pathology, 72, 141-148.

Grotte, G. (1956). Passage of dextran molecules across the blood-lymph barrier. Acta Chirurgica Scandinavica, Suppl., 211.

Koshurnikova, N. A., Aristov, V. P., Lemberg, V. K., Mushkasheva, G. A., and Popylko, M. G. (1973). Pathogenis of plutonium pneumosclerosis. Arkhiv Patologii, 35, (4), 48-54.

Koshurnikova, N. A., Aristov, V. P., Lemberg, V. K., Mushkacheva, G. A., Popylko, M. G., and Tseveleva, I. A. (1972). Mechanism of development of plutoniuminduced pulmonary sclerosis. Health Physics, 22, 753-754.,

Löbe, J., and Schneider, H. G. (1967). Untersuchungen zur enteralen $\mathrm{Cr}^{51}$ Albumin-Ausschreidung bei Abdominalbestrahlungen. Radiobiologia, Radiotherapia, 8, 47-50.

Loehry, C A., Axon, A. T. R., Hilton, P. J., Hider, R. C., and Creamer, B. (1970). Permeability of the small intestine to substances of different molecular weight. Gut, 11, 466470.

Madrazo, A., Suzuki, Y., and Churg, J. (1969). Radiation nephritis: acute changes following high dose of radiation. American Journal of Pathology, 54, 507-527

Majno, G., Gilmore, V., and Leventhal, M. (1967). On the mechanism of vascular leakage caused by histamine type mediators. A microscopic study in vivo. Circulation Research, 21, 833-847.

Majno, G., and Palade, G. E. (1961) Studies on inflammation. I. The effect of histamine and serotonin on vascular permeability: on electron microscopic study. Journal of Biophysics Biochemistry and Cytology, 11, 571-605.

Majno, G., Palade, G. E., and Schoefi, G. I. (1961). Studies on inflammation. I The site of action of histamine and serotonin along the vascular tree: a topographic study. Journal of Biophysics Biochemistry and Cytology, 11, 607-626.

Mathan, M., Mathan, V. I., and Baker, S. J. (1975). An electron-microscopic study of jejunal mucosal morphology in control subjects and in patients with tropical sprue in Southern India Gastroenterology, 68, 17-32

Mayerson, H. S., Wolfram, G. G., Shirley, H. H., and Wasserman, K. (1960). Regional differences in capillary permeability. American Journal of Physiology, 198, 155-160

Pappenheimer, J. R., Renkin, E. M., and Borrero, L. M. (1951). Filtration, diffusion and molecular sieving through peripheral capillary membranes. A contribution to the pore theory of capillary permeability. American Journal of Physiology, 167, 13-46.

Shiner, M. (1967). Ultrastructure of jejunal surface epithelium in untreated idiopathic steatorrhoea. British Medical Bulletin, 23, 223-225.

Sullivan, M. F. (1961). Intestinal vascular permeability changes induced by radiation or nitrogen mustard. American Journal of Physiology, 201, 951-954.

Trier, J. S., and Browning, T. H. (1966). Morphologic response of the mucosa of human small intestine to $\mathrm{X}$-ray exposure. Journal of Clinical Investigation, 45, 194-204.

Vatistas, S., and Hornsey, S. (1966). Radiation-induced protein loss into the gastrointestinal tract. British Journal of Radiology, 39, 547-550.

Waldmann, T. A. (1972). Protein-losing enteropathy and kinetic studies of plasma protein metabolism. Seminars in Nuclear Medicine, 2, 251-264.

Weinstein, W. M., Shimoda, S. S., Brow, J. R., and Rubin, 
C. E. (1970). What is celiac sprue? In Coeliac Disease, pp. 232-243. Edited by C. C. Booth and R. H. Dowling. Churchill Livingstone: Edinburgh.

Wiernick, G., and Plant, M. (1970). Radiation effects on the human intestinal mucosa. Current Topics in Radiation Research, VII, 325-368.

Willoughby, D. A. (1962). Evidence for a possible role of endogenous catecholamines in the vascular changes produced in rat intestine by X-rays. Journal of Pathology and Bacteriology, 83, 389-397.

Willoughby, D. A. (1960). Pharmacological aspects of the vascular permeability changes in the rat's intestine following abdominal radiation. British Journal of Radiology, 33, 515-519.

Willoughby, D. A. (1961). The effect of DFP on the acute intestinal radiation syndrome in the rat. British Journal of Radiology, 34, 807-813.

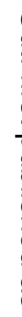

